\title{
Interoperability Test Suite Derivation for the TCP
}

\author{
Soonuk Seol ${ }^{\dagger}$, Myungchul Kim ${ }^{\dagger}$, Sungwon Kang ${ }^{\dagger \dagger}$, Yongbum Park ${ }^{\dagger, *}$, and \\ Younghan Choe ${ }^{*}$ \\ suseol@icu.ac.kr,mckim@icu.ac.kr,kangsw@sava.kotel.co.kr,ybpark@pec.etri.re.kr, and \\ yhchoe@pec.etri.re.kr \\ ${ }^{\dagger}$ Information and Communications University: ${ }^{\dagger \dagger}$ Korea Telecom Research \& Development \\ Group: ${ }^{*}$ Electronics and Telecommunications Research Institute
}

\begin{abstract}
A test derivation method suitable for testing interoperability for the class of communication protocols like the ATM/B-ISDN signaling protocol was proposed in [KanK 97]. Up to now, there is no work implementing automatic derivation of the interoperability test suite in the method and analyzing its coverage. In this paper, we implement the algorithm proposed in [KanK 97], apply it to the ATM/B-ISDN signaling protocol and the TCP protocol, and analyze the application results. In this process, we (1) improve the algorithm so that it can handle features like the TCP's three-way handshaking, (2) implement the improved algorithm as a program and derive an interoperability test suite automatically as a result of applying it to a pair of communicating TCP FSMs, and (3) analyze the generated interoperability test suite in terms of transition coverage'.
\end{abstract}

\section{Keywords}

protocol testing, interoperability testing, conformance testing, test architecture, test suite generation, transition coverage, ATM/B-ISDN, TCP, SDL

\section{INTRODUCTION}

To increase the confidence in protocol products implemented according to international standards, various protocol testing methodologies have been developed and applied. As an activity, conformance testing that checks whether an implementation is correct with respect to the relevant standards has been standardized by ISO [ISO 94] and applied to protocols such as $\mathrm{N}$ ISDN, ATM/B-ISDN, and so on. Nevertheless, it is widely known that conformance testing has limitation in ensuring interoperability. Thus, even a pair of two conforming implementations can fail to interoperate [RafC 90, APRS 93]. Two main causes of non-interoperation of conforming

\footnotetext{
${ }^{1}$ Transition coverage is the proportion of executed transitions over the whole transitions after validating or simulating a system.
} 
implementations are ambiguity of protocol standards and incompleteness of conformance testing [ShiK 98]. There is some research work on interoperability testing done in the past. [RafC 90] deals with interoperability test suite generation based on reachability analysis. However, it is not based on a rigorous definition of interoperability and considers only the case where lower testers exist between two IUTs. [VerB 94] describes experiences with interoperability testing of the FTAM protocol that uses only a single tester between two IUTs and thus has limited capability. [APRS 93] derives conformance test suite and interoperability test suite separately and later manually combines them to reduce the number of conformance test cases. [KanK 97] develops a method for systematically dealing with symmetric communication protocols ${ }^{2}$ and uses an interoperability test architecture that does not observe the interface behavior between two IUTs. [ShiK 98] proposes and applies a test derivation method suitable for testing interoperability for the class of communication protocols like the ATM/BISDN signaling protocol. Comparing the work listed above, our work made contributions to the automatic derivation of interoperability test suites and test coverage analysis of its applications results. The two papers [KanK 97, ShiK 98], which are closely related to our work, derive interoperability test suite manually and stop with applying their methods to the ATM/B-ISDN protocol. As a step to improve the method, we enhance and implement the algorithm. Then we apply the implemented program not only to the ATM/BISDN signaling protocol but also to TCP protocol, and analyze the application results. In addition, we compare and analyze transition coverage of two cases: one case carried out by applying the validation in the commercial SDL tool [SDT 97] to the IUT and the other case carried out by applying the interoperability test suite utilizing the simulation facility of the commercial tool.

This paper is organized as follows: In Section 2, we state backgrounds related to this work. Section 3 introduces our enhanced algorithm by analyzing the problems in the existing algorithm. In Section 4, we apply the algorithm to the ATM/B-ISDN signaling protocol and the TCP protocol. During this process, we generate the interoperability test suites both for the ATM/B-ISDN protocol and the TCP. In Section 5, we compare transition coverages of two cases: one obtained from applying the interoperability test suite derived in Section 4 to the simulator of the commercial SDL tool and

${ }^{2}$ When implementations based on the same protocol specification interact each other, we define the protocol as symmetric protocol (e.g., ATM/B-ISDN signaling protocol and TCP). If protocol specifications which have implemented as IUTs are different (e.g., server/client), we define the protocol as asymmetric protocol. 
the other from validation process using the validator of the tool. Finally in Section 6, we discuss the conclusion and the future work.

\section{BACKGROUND}

It is difficult to stimulate/observe the interactions between processes or systems because of the asynchronicity in concurrent programs or distributed systems. To avoid these difficulties, [AraS 92] imposes two principles on the black-box testing for concurrent programs. First, scheduler-independent observation principle makes events arise from each process without influence of scheduler algorithm. In many cases, this principle implies that the observation cannot distinguish the order of events occurring in different processes. Second, single stimulus principle means that a single stimulus must be given at each stable state. A stable state for concurrent programs is defined in [AraS 92] as the system state (1) that is reachable from the initial state adhering to the single stimulus principle, and (2) from which no change can occur without another stimulus. The system adhering to the single stimulus is considered as the system running in a slow environment [LuBP 94]. In a slow environment, inputs can be sent from the environment to the system only in situations where all the queues and all the channels are empty. According to the single stimulus principle, multiple stimuli and stimuli during transitions are not considered. The interoperability test suite derivation algorithm proposed by [KanK 97, ShiK 98] is based on single stimulus principle that is the viewpoint of regarding the response of implementations for a single input as constituting a test case.

[ShiK 98] presents three test architectures for the interoperability testing. In this paper, only two of them are considered. They are shown in Figure 1. In Test Architecture I, Tester A sends a message to IUT A and then IUT A can sends messages to IUT B and/or Tester A. If IUT B receives the message, it can also sends messages to IUT A and/or Tester B. Tester $C$ located between IUT A and IUT B can observe/modify messages between IUT A and IUT B. In Test Architecture II, however, it is impossible to read these messages owing to absence of Tester C.

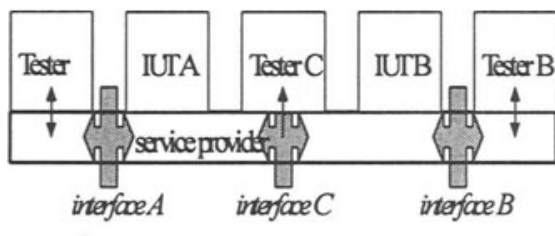

$<$ TestArchitectureP

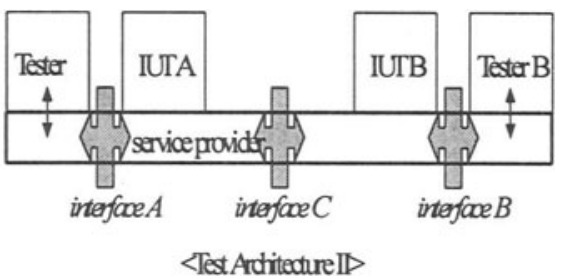

$<$ TestArditectureI $>$

Figure 1. Interoperability test architectures. 
To achieve interoperability testing, a composite Finite State Machine (FSM) need be derived. Let us represent a protocol specification with an FSM in order to formally describe the derivation process of composite FSM. Let M be the FSM for the protocol specification of an IUT. Then $M$ can be represented as follows:

Definition 1. A FSM is a 5-tuple $\left(S, S_{0}, L_{\text {in }}, L_{\text {out }}, T r\right)$ where:

(1) $S=\left\{S_{0}, S_{1}, \ldots, S_{n-1}\right\}$ is a set of states of $M$,

(2) $S_{0} \in S$ is the initial state,

(3) $\mathbf{L}_{\text {in }}=\left\{v_{1}, v_{2}, \ldots, v_{m}\right\}$ is a set of input symbols,

(4) $\mathbf{L}_{\text {out }}=\left\{u_{1}, u_{2}, \ldots, u_{k}\right\}$ is a set of output symbols, and

(5) $\operatorname{Tr} \subseteq\left\{S-v / U \rightarrow S^{\prime} \mid S, S^{\prime} \in S \wedge v \in L_{\text {in }} \wedge U \subseteq \mathbf{L}_{\text {out }}\right\}$ is a set of transitions.

Bold letters in Definition 1 represent sets. " $S-v / U \rightarrow S^{\prime}$ " stands for a transition where $S, S^{\prime}, v$, and $U$ are, respectively, the starting state, the destination state, an input symbol and a set of output symbols.

Let $\Pi$ be the composition of the two FSMs, one, $M_{A}$, for the specification A and the other, $M_{B}$, for the specification $B$. We assume that $U$ in Definition 1, for both $M_{A}$ and $M_{B}$, consists of at most two output symbols where each symbol is for different interface. Then $\Pi$ can be defined as follows:

Definition 2. The composite FSM $\Pi$ is a 5-tuple $\left(\mathbf{S}_{\Pi}, S_{\Pi, 0}, \mathbf{L}_{\Pi, \text { in }}, \mathbf{L}_{\Pi, \text { out }}, \mathbf{T r}_{\Pi}\right)$ where:

(1) $S_{\Pi}=\left\{S_{\Pi, 0}, S_{\Pi, 1}, \ldots, S_{\Pi, n-1}\right\}$ is a set of global states, and $S_{\Pi, i}=\left(S_{A}, S_{B}\right)$, where $S_{A}$ and $S_{B}$ are states of $M_{A}$ and $M_{B}$ respectively.

(2) $S_{\Pi, 0} \in S_{\Pi}$ is the initial state. Namely, $S_{\Pi, 0}=\left(S_{A, 0}, S_{B, 0}\right)$.

(3) $\mathbf{L}_{\Pi, \text { in }}=\left\{\mathbf{v}_{\Pi 1}, \mathbf{v}_{\Pi 2}, \ldots, \mathbf{v}_{\Pi \mathrm{m}}\right\}$ is a set of input symbols. It consists of input messages for the interface $A$ and $B$. These messages are external inputs from testers. Namely, $\mathbf{L}_{\prod, \text { in }}=\left(\mathbf{L}_{\mathrm{in}, \mathbf{A}, \mathbf{E}} \cup \mathbf{L}_{\mathrm{in,B,E}, \mathrm{E}}\right)$.

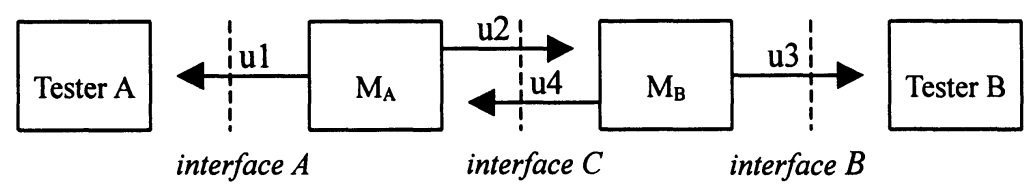

Figure 2. Output Symbols of two FSMs.

(4) $\mathbf{L}_{\Pi, \text { out }}=\left\{U_{\Pi 1}, U_{\Pi 2}, \ldots, U_{\Pi \mathbf{k}}\right\}$ is a set of output symbols. As shown in Figure 2, $U_{\Pi i}$, an element of $L_{\Pi, \text { out }}$, is represented as vector $<u 1, u 2, u 3$, $u 4>$, where $u 1, u 2, u 3$, and $u 4$ represent messages transmitted by $M_{A}$ via 
interface $A, M_{A}$ via interface $C, M_{B}$ via interface $B$, and by $M_{B}$ via interface $C$ respectively.

(5) $\mathbf{T r}_{\Pi} \subseteq\left\{S_{\Pi}-v_{\Pi} / U_{\Pi} \rightarrow S_{\Pi}^{\prime} \mid S_{\Pi}, S_{\Pi}{ }^{\prime} \in S_{\Pi} \wedge v_{\Pi} \in L_{\Pi \text {,in }} \wedge U_{\Pi} \in L_{\Pi, \text { out }}\right\}$ is a set of transitions.

Note that set of transitions $\mathbf{T r}_{\Pi}$ derived by the interoperability test suite derivation algorithm [KanK 97, ShiK 98] includes not only interoperability test cases but also conformance test cases.

\section{ENHANCEMENT OF THE INTEROPERABILITY TEST SUITE DERIVATION ALGORITHM}

In this section, we propose our enhanced algorithm by analyzing problems in the algorithm shown in [ShiK 98].

\subsection{Problems in the Existing Algorithm}

The existing algorithm proposed by [ShiK 98] has a problem that the algorithm is not applicable to a case where there are more than two messages between two IUTs for an external input. Namely, the number of internal messages caused by an external input are limited to two. These internal messages such as $\mathrm{u} 2$ and $\mathrm{u} 4$ in Figure 2 do not interact with the environment.

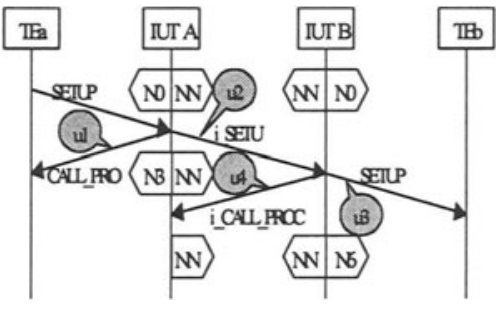

Figure 3. An example of message interaction

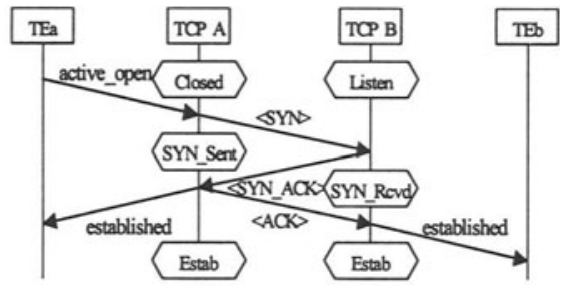

Figure 4. An example of message interaction for the ATM/B-ISDN signaling protocol for the TCP.

In the case of ATM/B-ISDN signaling protocol as shown in Figure 3, internal messages between two IUTs can be represented with two elements (i.e., u2 and u4) in a vector $<\mathrm{u} 1, \mathrm{u} 2, \mathrm{u} 3, \mathrm{u} 4>$ defined in Definition 2 - (4).

In general protocols, however, internal messages can not be represented with this data structure when the number of messages exchanged between two IUTs is more than two. For instance, TCP connection is made with a threeway handshake. So the three messages, SYN, SYN_ACK, and ACK occur at the interface $\mathrm{C}$ located between TCP A and TCP B as shown in Figure 4. These messages can not be represented only with $u 2$ and $u 4$. 


\subsection{The Enhanced Algorithm}

We solve the problem pointed out in the previous section by redefining the Definition $2-(4)$ as follows:

\section{Definition 2'.}

(1) $\mathrm{W}_{\Pi}=\left[\mathrm{U}_{\Pi, 1} \oplus \ldots \oplus \mathrm{U}_{\Pi, i} \oplus \ldots \oplus \mathrm{U}_{\Pi, l}\right]$ is the concatenation of output symbols sequence for an external input. The output symbols $U_{\Pi, i}$ is represented by the vector $\langle\mathrm{u} 1, \mathrm{u} 2, \mathrm{u} 3, \mathrm{u} 4>$ where $\mathrm{u} 1, \mathrm{u} 2, \mathrm{u} 3$, and $\mathrm{u} 4$ represent messages transmitted by $M_{A}$ via interface $A, M_{A}$ via interface $C, M_{B}$ via interface $B$, and by $M_{B}$ via interface $C$ respectively.

(2) $\mathbf{L}_{\Pi, \text { out }}=\left\{\mathrm{W}_{\Pi 1}, \mathrm{~W}_{\Pi 2}, \ldots, \mathrm{W}_{\Pi k}\right\}$ is a set of output symbol sequences.

The following is an enhanced algorithm adhering to the Definition $2^{\prime}$. The statements in the dashed box are the part of original algorithm [ShiK 98], and the statements appended or modified from the algorithm are represented in shadow.

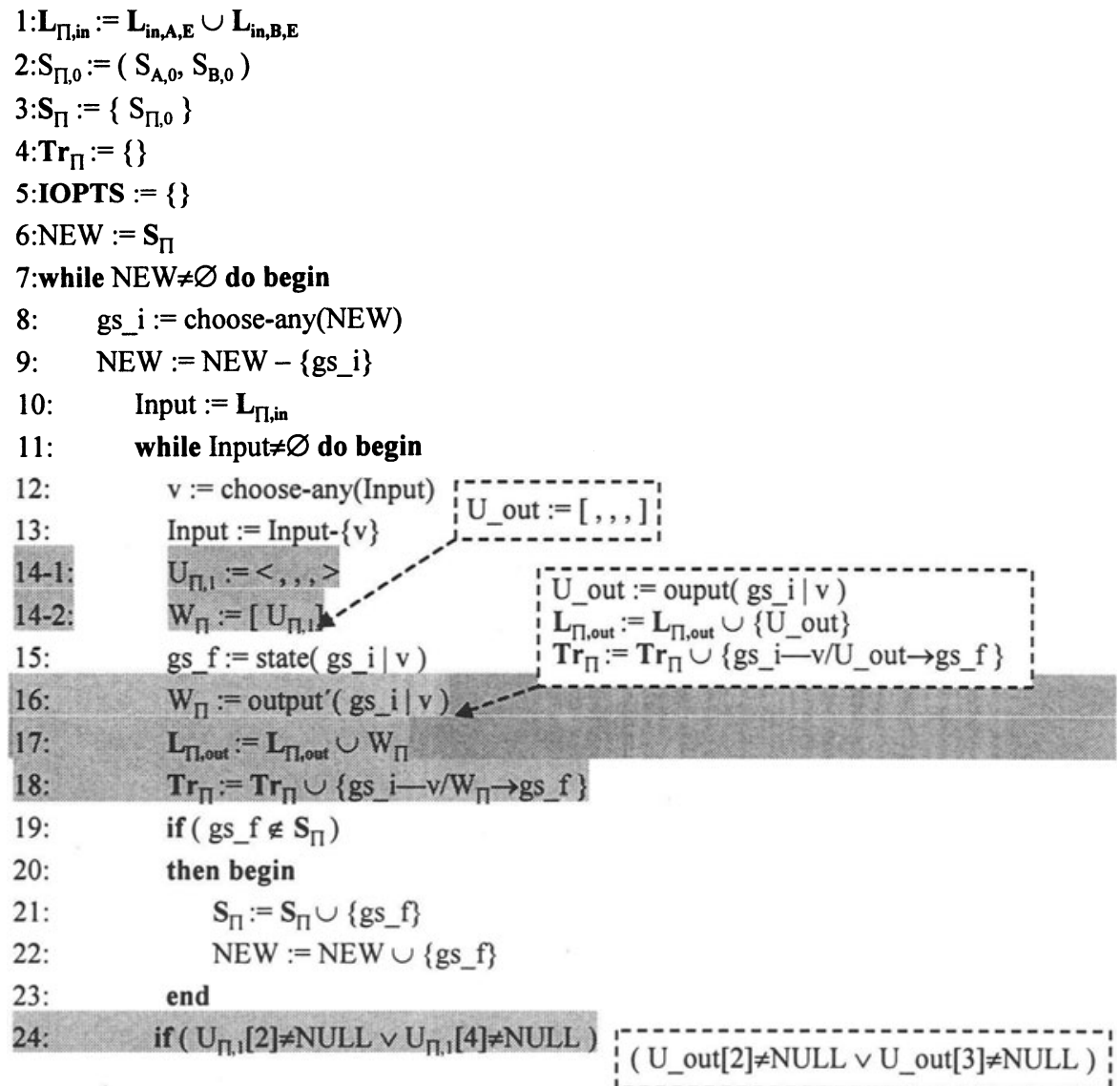




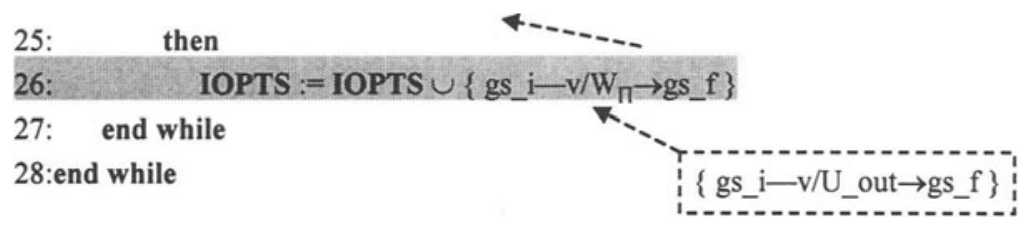

Algorithm 1. Enhanced algorithm for interoperability test suite derivation.

Now we confirm that the enhanced algorithm is applicable to the case in Figure 4. In line 1 of the Algorithm 1 , a set of input symbols $\mathbf{L}_{\Pi \text {,in }}$ is initialized by external inputs such as passive_open, close, send_data, and active_open which were shown in TCP FSM. By initialization steps from line 1 to 6, an initial value of the set $N E W$ becomes the initial state (Closed, Closed). To explain an example in Figure 4, we assume that the start stable state (Closed, Listen) has been already added to the set $N E W$ as a result of progress of the algorithm. Also, we assume that start stable state gs_ $i$ gets state (Closed, Listen) from the set $N E W$ in line 8 and the input symbol $v$ gets the input symbol active_open from a set of input symbols Input in line 12 . Then, $\mathrm{W}_{\Pi}$ becomes [ $<$, SYN, SYN_ACK $>$, < established, ACK, established, > ] by the function output' () in line 16 after it is initialized in line 14. These output symbols can not be represented with the existing algorithm's data structure $U_{\Pi}$ (i.e., $<u 1, u 2$, u3, u4>). Figure 5 summarizes the proceeding of the algorithm.

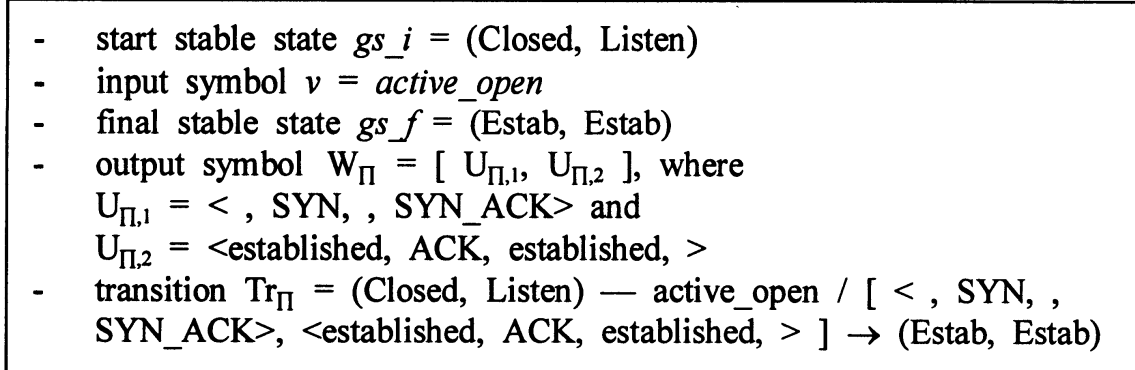

Figure 5. Application of Algorithm 1 to the example in Figure 4.

Our enhanced algorithm is the generalized one which is applicable to protocols in general since the algorithm have no limitation on the number of internal messages.

The program implementing Algorithm 1 gets FSMs corresponding to two IUTs as a file, executes the data in the file, and writes its result (namely, interoperability test suite) to a file like the format in Appendixes 1 and 2 . The time taken for generating each test suites is negligible. 


\section{INTEROPERABILITY TEST SUITE DERIVATION}

In this section, we demonstrate the feasibility of Algorithm 1 in Section 3 by applying the implementation of the algorithm to ATM/B-ISDN signaling protocol used in [ShiK 98]. Then we show the generality of this algorithm through derivation of interoperability test suite for connection management (establishment/release) part of TCP.

\subsection{Application to ATM/B-ISDN Signaling Protocol}

The result of applying the implementation of the enhanced algorithm to ATM/B-ISDN signaling protocol is described in Appendix 1. Out of the thirty-two test cases derived, twenty-six test cases are for interoperability testing and six test cases are for conformance testing. The result of this application is the same as that of the interoperability test suite derived manually in [ShiK 98]. The test cases for conformance testing in Appendix 1 were not given in [ShiK 98].

Let us explain an example of interoperability test case corresponding to the scenario in Figure 3. The test case is equivalent to item (1) of Appendix 1 and is as follows:

$(0: 0,0: 0)$-- SETUPa/[ <CALL_PROC,i_SETUP,SETUP,i_CALL_PROC $>$ ] $\rightarrow(3: 9,6: 3)$

An input symbol with postfix ' $a$ ' (or ' $b$ ') represents a message transmitted by tester TEa (or TEb) via interface A (or interface B). ' $i$ ', is used to indicate internal messages as opposed to external messages. Each element of the starting stable state $(0: 0,0: 0)$ denotes state N0:NN0 of IUTa and state $\mathrm{N} 0$ :NN0 of IUTb, respectively. SETUPa before ' $/$ ' is an input symbol to IUTa and [ <CALL_PROC, i_SETUP, SETUP, i_CALL_PROC> ] after ' $/$ ' is output symbol sequence.

\subsection{Application to TCP}

In this section we demonstrate the generality of this algorithm by applying it to TCP. In this paper, we consider only the connection management part of TCP.

\subsubsection{Modeling TCP interoperability testing architecture}

Testers A and B of test architectures in Figure 1 correspond to Lower Testers for IUT A and IUT B, respectively. To test interoperability of TCP we need to modify Test Architecture II. The modified test architecture is in Figure 6. It has two Upper Testers that are able to access user interfaces of TCP A and TCP B, respectively. In spite of this modification, the same algorithm can be used to derive interoperability test suite. In case that we apply the algorithm 
to derive interoperability test suites from the point of view of the users of the interacting protocols or systems, the method can be utilized for service testing which tests service interoperability of protocols or systems under test. Upper Tester controls and observes TCP through Point of Control and Observation (PCO). For example, in the case of socket communication, Upper Tester makes use of Abstract Service Primitives (ASPs) such as socket, bind, listen, accept, and connect to control and observe TCP. Two testers may be merged in a local test environment whereas testers may be designed to communicate with each other through a separate communication channel in a distributed test environment.

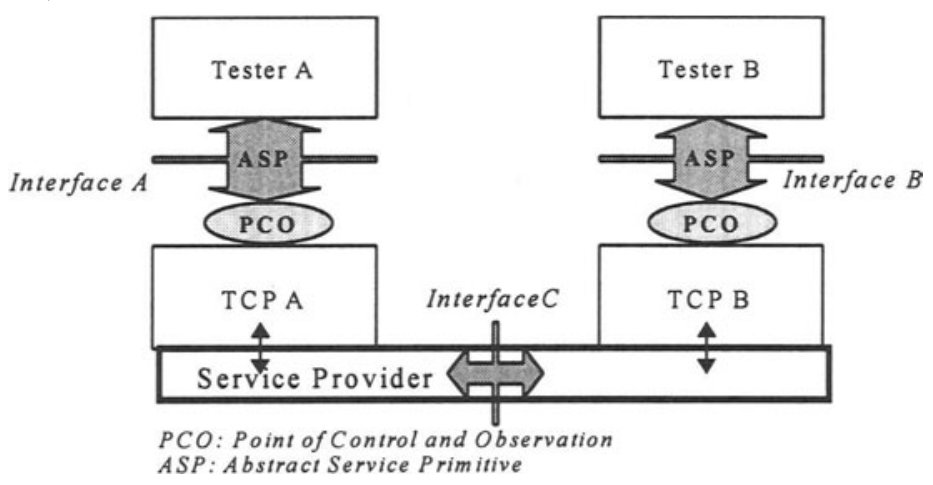

Figure 6. Architecture for TCP interoperability.

\subsubsection{FSM representation of TCP}

Figure 7 is an FSM for connection management part of TCP [GarR 95]. The FSM has eleven states and describes two procedures: one for connection establishment from state CLOSED to state ESTABLISHED and the other for connection release. Data transfer procedure at state ESTABLISHED is not dealt with here. Note that the FSM has simultaneous open and simultaneous close procedures. When both systems actively ask for a connection simultaneously, the procedure is called simultaneous open or simultaneous connection establishment. A simultaneous close occurs when both applications initiate a close at the same time. Input is given by the upper application program running on TCP and output is presented by one or more bit selection among URG, ACK, PSH, RST, SYN, and FIN in the 6-bit control field of TCP packet. TCP controls connection management by selection of these bits. 


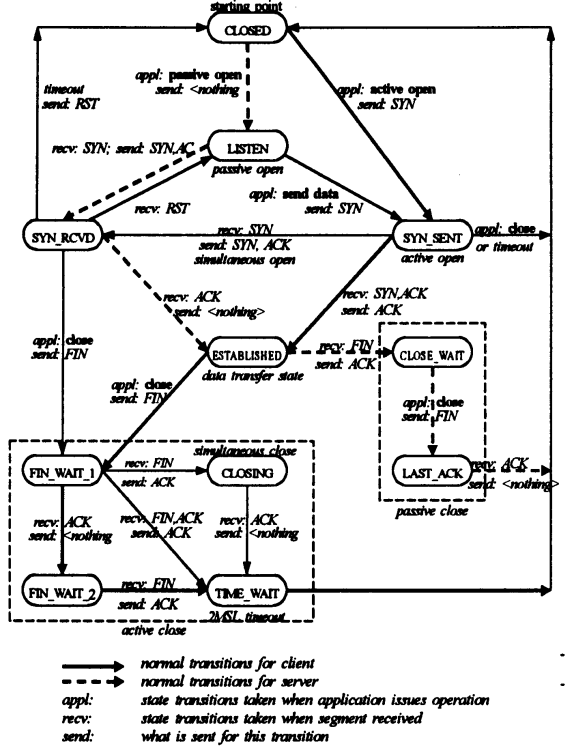

Figure 7. Simplified TCP FSM.

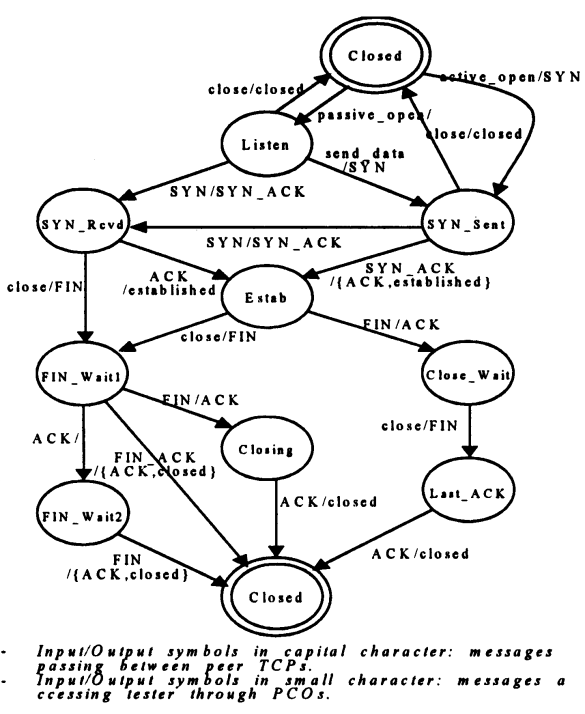

Figure 8. TCP FSM

.In this paper, we make use of the FSM in Figure 8 obtained by omitting the behavior related to timer from Figure 7. The reason for the omission is that the tester cannot control the internal behavior of IUT relating to time. This omission makes it impossible to test RST message handling the part that invokes connection re-establishment. In the FSM, there are three types of messages: messages from tester to TCP, messages between two TCPs represented in bold characters, and messages (which are responses of socket communications) from TCP to the tester through PCO. The last type messages are extracted from the basic socket system calls [Rich 91].

\subsubsection{Application result}

The application result of the implementation of the enhanced algorithm to the connection management part of TCP is described in Appendix 2. Out of the thirty-two test cases derived, sixteen test cases are for interoperability testing and sixteen test cases for conformance testing. The ratio of the number of the conformance test cases over that of the interoperability ones for TCP is higher than that of ATM/B-ISDN signaling protocol because of the fact that there are several paths for connection establishment in TCP. Namely TCP has a negotiation procedure returning to the initial state when there is some disagreement between peer TCPs. For example, TCP FSM in Figure 8 returns to state Closed if IUT does not receive a proper message after it transits to state SYN_Sent by receiving an active_open message from state Closed. TCP establishes its connection via passing through states Listen or SYN_Sent from the initial state whereas ATM/B-ISDN signaling protocol establishes its connection by a single SETUP message. Since TCP has local 
behavior without any message exchange with peer TCP as the above, more conformance test cases are needed.

Let us show an example of test case for the message interaction in Figure 4. This test case is equivalent to item (13) of Appendix 2 and as follows:

(Closed,Listen) -- active_open_a/[ <,i_SYN, ,i_SYN_ACK >, <established, i_ACK, established,> ] $\rightarrow$ (Estab,Estab)

\section{TRANSITION COVERAGE}

In this section we investigate transition coverage of the TCP interoperability test suite derived in Section 4. For this, we view the TCP FSM simulator as the IUT and carry out two case studies of transition coverage: one for that of the derived interoperability test suite and the other for that of validation by a commercial SDL tool. In the latter case, if there are no errors in the specification and its SDL model, we should get $100 \%$ coverage provided that the validation method of choice is complete. We first explain the two cases and then we compare their respective transition coverage. Also we analyze the reasons why certain transitions are not covered.

\subsection{Two Case Studies of Transition Coverage Calculation}

This section conducts two case studies of calculating transition coverage. The first one (Case I) is carried out by applying the commercial SDL tool to the IUT. Let us denote by TC1 the transition coverage of this case. The second case (Case II) is carried out by applying the interoperability test suite utilizing the simulation facility of the commercial tool. Let us denote by TC2 the transition coverage of this case. Since Case I is for validation and Case II is for interoperability testing, their purposes are different. However, it is meaningful to compare TC2 with TC1 because the TC1 is obtained by exercising mechanically and completely all the transitions in a step by step manner.

Case I is carried out in Bit-State mode which examines all possible transitions for each state. The Bit-state mode is a complete validation method. Case I is carried out under the validation architecture of Figure 9. This structure is designed such that input message to the two IUTs are applies from the external environment. When the Bit-State mode is used, this external input should be selected automatically by the commercial tool.

In Case I, the validation tool controls the external environment whereas in Case II the tester controls it. Thus as shown in Figure 10 the test architecture for Case II is designed such that the tester communicates with the external environment of the validation architecture. Moreover, since Case II is for 
interoperability testing, in order to apply one or more interoperability test cases, each test case should start in the initial state. For this, IUT' used in the test architecture of Case II is augmented with transitions that take us to the initial state upon receiving reset message in each state.

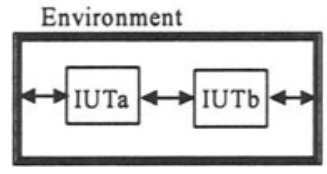

Figure 9. Validation Architecture.

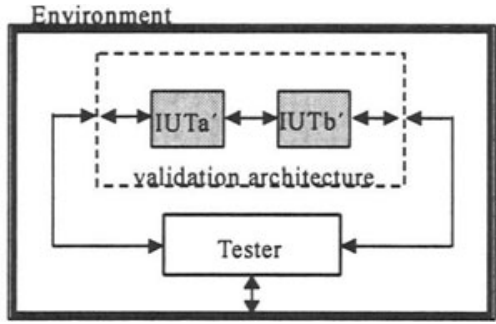

Figure 10. Test Architecture.

Since the test architecture of Case II follows the test architecture suggested in Figure 6, it requires two testers. But we assume that one tester performs the role of two testers and the underlying layers are reliable and correct and consider only Protocol Data Unit (PDU) for TCP.

Since Case II is for interoperability testing, Case II comparing with Case I has the following properties:

P1. There are transitions from any state to the initial state when the IUT receives reset signal from the tester. Uncovered transitions out of those transition executed by reset signal should be excluded in calculating transition coverage because those ones are added for testing purpose.

P2. The test cases are for interoperability testing. Thus, uncovered transition out of those transitions executed for conformance testing should be excluded in calculating the transition coverage of the interoperability testing.

\subsection{The Comparison Result}

Figure 11 depicts the SDL model of TCP FSM shown in Figure 8. The two target IUTs of interoperability testing works based on the same FSM. Therefore, we examine transition coverage with respect to one IUT's FSM only, that for the other one being the same. The total transition coverage for the whole system of IUTs is twice that of one IUT. 


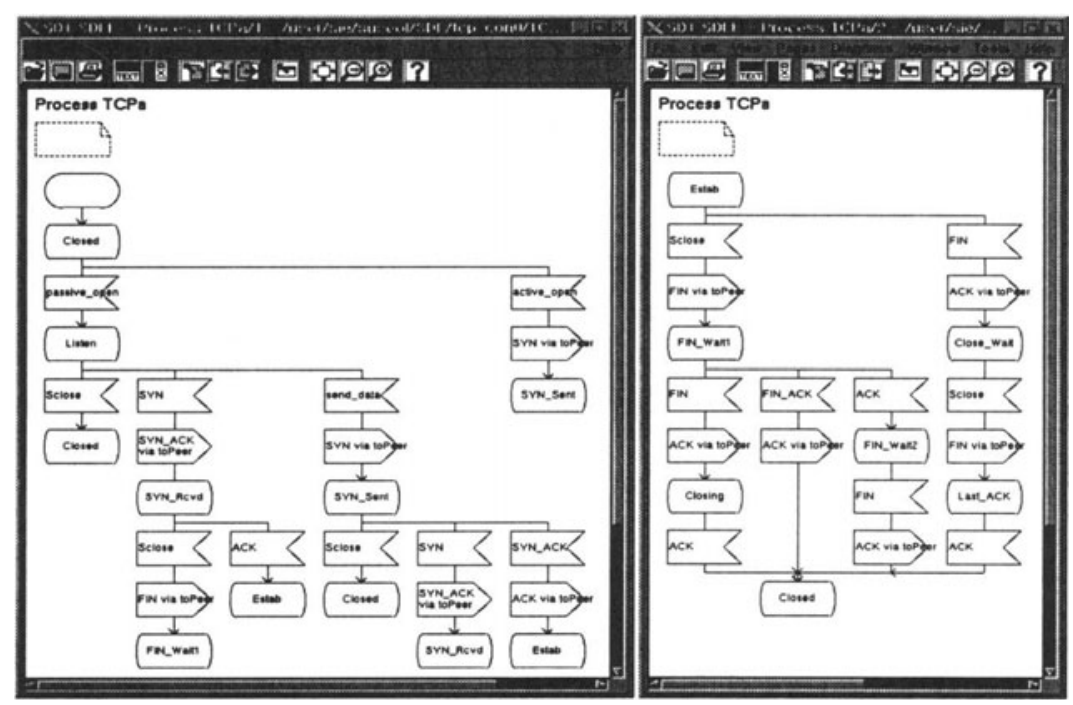

Figure 11. SDL Description? ${ }^{3}$.

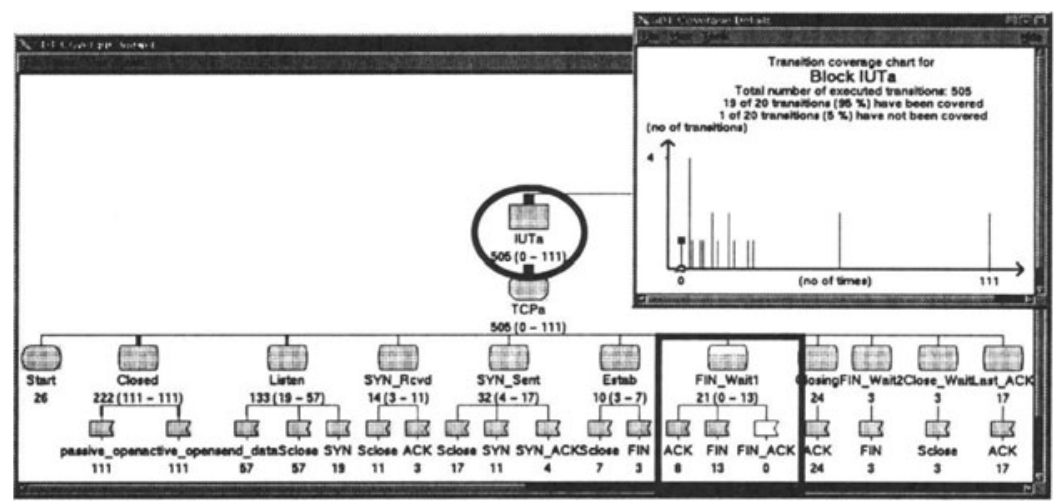

Figure 12. Transition coverage tree and chart for Case I.

The transition coverage for Case I obtained by validation under the architecture of Figure 9 is shown in Figure 12. The left-hand side figure of Figure 12 depicts the part for the IUTa among the whole transition coverage tree. The size of shaded parts indicates the ratio of the executed transitions. In order to illustrate the numbers, let us take the example of " $505(0-111)$ " in the circle, in which:

- 505 transition have been executed,

\footnotetext{
${ }^{3}$ The word 'close' is reserved in the commercial SDL tool. Thus we prefix it with ' $\mathrm{S}$ '.
} 
- the least frequently executed transition in block IUTa has been executed zero times, and

- the most frequently executed transition in block IUTa has been executed 111 times.

In the lower part of the tree, there is one transition that was never executed. It is represented by 0 in the rectangle of Figure 12. It is the transition that was not executed upon receiving FIN_ACK in FIN_Wait1 state. Note that there is no shade for the input symbol FIN_ACK.

Let us look at the transition coverage chart on the right-hand side of Figure 12. Total number of executed transitions is 505 , the number of transitions that are executed at least once is $19(95 \%)$ and that of transitions that are never executed is $1(5 \%)$. The horizontal axis indicates the number of executions of the transition under consideration. When this number is 0 , it means that the transition was never executed. The execution times of the most frequently executed transition are 111 . The vertical axis indicates the number of relevant transitions. The transition that was never executed is the transition from FIN_Waitl to Closed state among those marked with thick arrows in IUT FSM of Figure 14. sIn the TCP FSM ${ }^{4}$ of Figure 7 shown in a book [GarR 95], there are three paths from FIN_Waitl state to Closed state: (1) upon receiving FIN, move to Closing state and then move to Closed state upon receiving ACK, (2) upon receiving ACK, move to FIN_Wait2 state and then move to Closed state upon receiving FIN and (3) upon receiving FIN_ACK, move to Closed state. In the last case, FIN_ACK means that among the control bits of the packet both FIN bit and ACK bit are set. Even though there are three possibilities, there is no state sending FIN_ACK corresponding to (3) and therefore this transition cannot be executed. That is, the protocol specification itself is not complete. Thus the transition coverage would be $100 \%$ if we do not consider the transition.

Let us examine the transition coverage for Case II. Here we want to calculate the test coverage of the interoperability test suite derived using the program realizing Algorithm 1. It is shown in Figure 13.

The left-hand side of Figure 13 depicts only the part with $0 \%$ coverage for IUTa among the whole transition coverage tree. As shown, there are 13 transitions in IUTa that are never executed. The chart in the right-hand side of Figure 13 represents that total number of executed transitions is 41 , and $17(57 \%)$ transitions are covered and $13(43 \%)$ ones are not covered out of the total 30 transitions. Note that 10 transitions triggering by reset input out of the total 30 ones were added for testing purpose (See property P1 in Section 5.1). The 10 transitions consist of 7 ones out of the 13 uncovered

${ }^{4}$ In the TCP FSM of RFC [RFC 81], on which the FSM of [GarR 95] is based, there is no transition to take from FIN_Waitl state to Closed state upon receiving FIN_ACK. 
ones and 3 ones out of the 17 covered ones (Those data are in columns $T_{\text {reset }}$ and Total in Table 1). Considering transitions corresponding with the total 20 transitions of the TCP specification which was dealt with in Case I, the number of uncovered transitions is 6 (which is equivalent to the sum of corresponding $\mathrm{T}_{\text {IOPT }}$ and $\mathrm{T}_{\mathrm{CT}}$ in Table 1 ) and these transitions are marked with thick arrows in Figure 14.

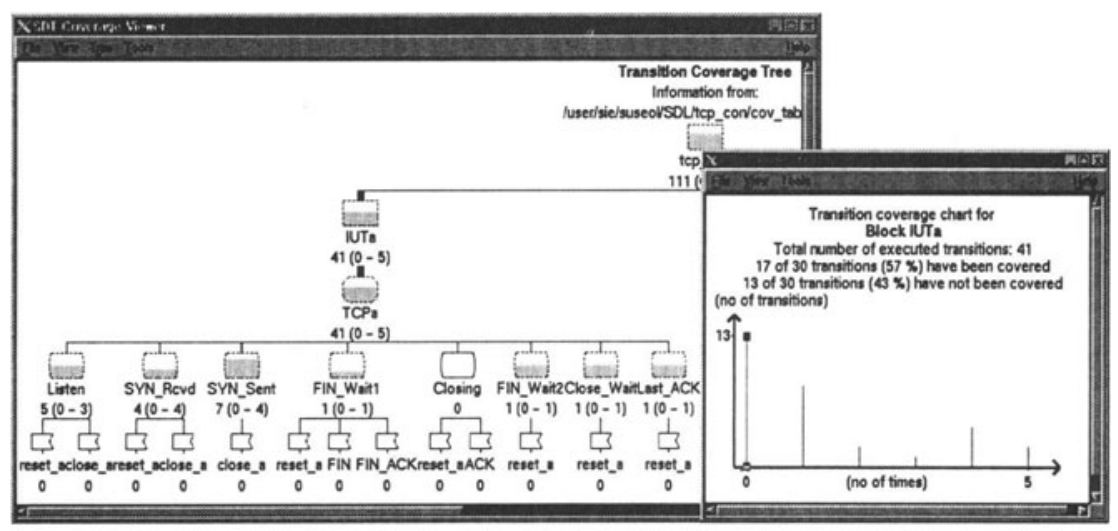

Figure 13. Transition coverage tree and chart for Case II.

\subsection{Result Analysis}

Transitions need to be classified in order to analyze the transition coverage of Case II.

Definition 3. Transition classification

- $\mathrm{T}_{\mathrm{CT}}$ : those transitions not having messages passing through interface $\mathrm{C}$.

- $\mathrm{T}_{\mathrm{IOPT}}$ : those transitions having one or more than one messages passing through interface $\mathrm{C}$.

- $T_{\text {init }}:$ the transition to initialize FSM.

- $T_{\text {reset }}$ : those transitions moving from any state to the initial state by reset input.

According to Definition 3, we classify the transitions as Figure 15.

The following Table represents the transition coverage obtained by Case II.

Table 1. Transition coverage analysis on Case II

\begin{tabular}{ccccccc}
\hline Transition Classification & $\mathrm{T}_{\text {IOPT }}$ & $\mathrm{T}_{\mathrm{CT}}$ & $\mathrm{T}_{\text {init }}$ & $\mathrm{T}_{\text {reset }}$ & Total \\
The number of covered transitions & 12 & $(75 \%)$ & 1 & 1 & 3 & 17 \\
The number of uncovered transitions & 4 & $(25 \%)$ & 2 & 0 & 7 & 13 \\
Total & 16 & $(100 \%)$ & 3 & 1 & 10 & 30 \\
\hline
\end{tabular}




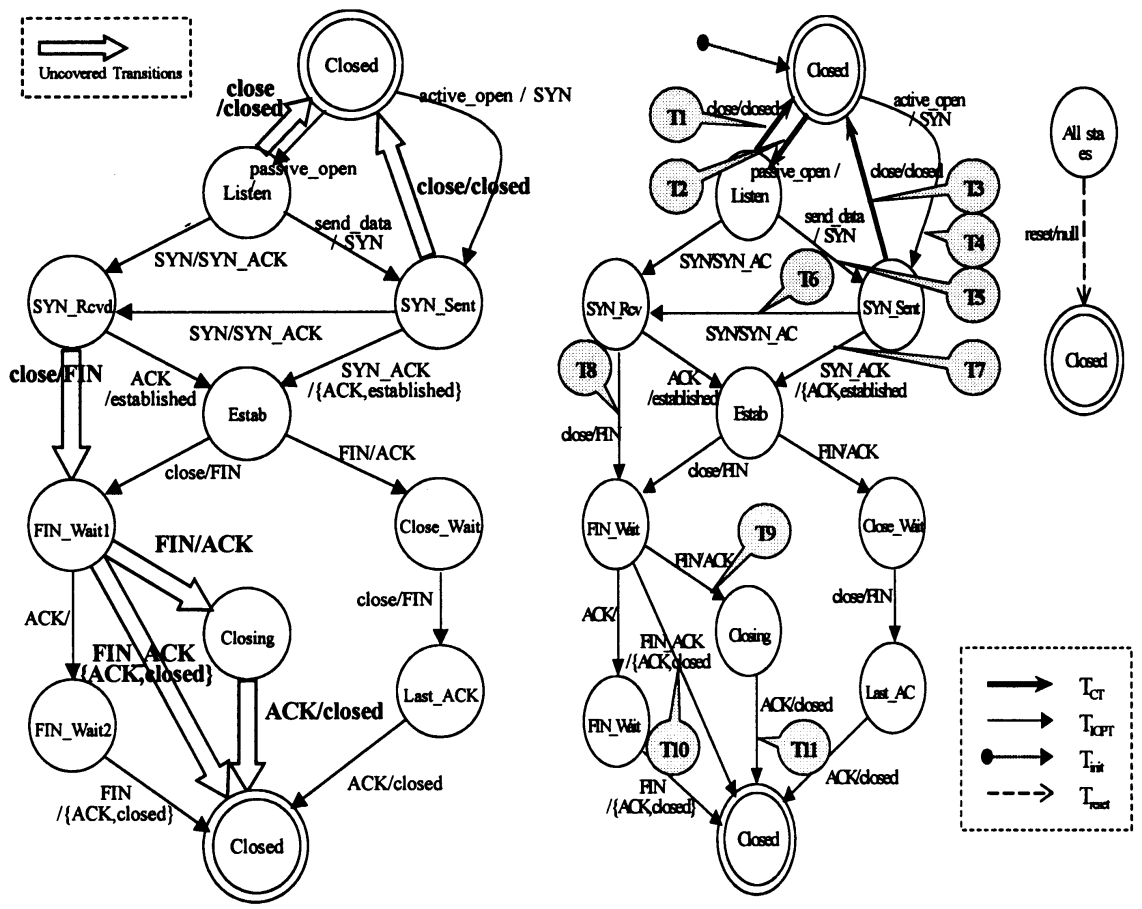

Figure 14. Uncovered transitions.

Figure 15. Transition classification.

Since $\mathrm{T}_{\mathrm{CT}}$ (i.e., $\mathrm{T} 1, \mathrm{~T} 2$, and $\mathrm{T} 3$ in Figure 15 ) corresponds to conformance testing, those transitions are not dealt with in Case II dealing with interoperability testing (See property P2 in Section 5.1). Note that one transition, $\mathrm{T} 2$, is executed during interoperability testing because the transition belongs to the preamble of some test cases for interoperability testing.

Let us analyze transition coverage of $\mathrm{T}_{\text {IOPT }}$. The uncovered transitions in $\mathrm{T}_{\text {IOPT }}$ are T8, T9, T10 and T11. Transition T10 is not covered because of incompleteness of the specification as mentioned before. Transitions T8, T9 and T11 are not executed because of the limitation of our algorithm (the limitation will be discussed briefly later in this section). Transition coverage of validation in Case I becomes $100 \%$ ( 15 transitions out of 15 ones in $\mathrm{T}_{\mathrm{IOPT}}$ ) if we exclude T10 which is not executed due to incompleteness of the specification. And transition coverage of our method applying interoperability test cases in Case II becomes $80 \%$ (12 transitions out of 15 ones in $\mathrm{T}_{\mathrm{IOPT}}$ ) if we exclude transition $\mathrm{T} 10$ as stated above. In addition, if we calculate the transition coverage including conformance testing, since all of three transitions in $\mathrm{T}_{\mathrm{CT}}$ in Table 1 can be executed, the transition coverage 
becomes $83 \%$ because of covering 15 transitions ( 12 transitions in $\mathrm{T}_{\mathrm{IOPT}}+3$ transitions in $\mathrm{T}_{\mathrm{CT}}$ ) out of 18 ones ( 15 in $\mathrm{T}_{\mathrm{IOPT}}+3$ in $\left.\mathrm{T}_{\mathrm{CT}}\right)$.

Total number of executed transitions to obtain $100 \%$ transition coverage in validation (Case I) is 505 whereas that of obtaining $80 \%$ in Case II is 41 . It is not possible to compare directly the number of executed transitions to obtain each transition coverage since those two cases are performed for different purposes. However, the data are very informative since interoperability testing is a kind of test exploring the total state space (which validation covers) with limited time and resource. As summarized in Table 2, it is worth deriving interoperability test cases having significant transition coverage spending relatively little time and resource.

Table 2. Transition coverage and cost.

\begin{tabular}{ccc}
\hline & Transition coverage & Number of executed transitions \\
Case I & $100 \%(15 / 15)$ & 505 \\
Case II & $80 \%(12 / 15)$ & 41 \\
\hline
\end{tabular}

Let us discuss briefly the limitation and possible extension of our algorithm which is further work. Transitions T8, T9 and T11 are not covered because of the single stimulus principle and test architecture which our algorithm is based on. Transitions T9 and T11 can not be executed because of the single stimulus principle since those transitions require two inputs at the same time. As the solution, we can adopt simultaneous stimulus principle meaning that two inputs can be applied to both IUTs at the same time. Since the order of events invoked by the simultaneous stimulus principle is nondeterministic, we may require stochastic approach to this control and observation. Also transition T8 can be performed in Test Architecture I (in Figure 1) having a tester between two IUTs. Since the tester is able to capture packets in transmission, packet loss can be simulated. By extending our method, those transitions can be covered and thus the transition coverage of interoperability test suite becomes $100 \%$ which is equivalent to that of validation.

\section{CONCLUSION AND FUTURE WORK}

In this paper, we demonstrated that the test suite derivation for the interoperability testing of protocols is possible efficiently and effectively in an automatic way. In order to do that, we enhanced the algorithm in [ShiK 98], implemented our enhanced algorithm as a program, and applied it to the ATM/B-ISDN signaling protocol and TCP protocol. As its results, we showed that our implementation of the enhanced algorithm generates the same interoperability test cases successfully with the test cases generated manually in [ShiK 98]. Also, we carried out an interoperability testing for the connection management part of the TCP by applying the derived test 
suite utilizing the simulation facility of the commercial SDL tool. The transition coverage in this case and the transition coverage when validation facility is utilized were compared and analyzed. As the result, except for the transition described ambiguously in the protocol specification, for obtaining $100 \%$ transition coverage in validation it took 505 transition execution whereas it took only 41 transition executions for obtaining $80 \%$ when we apply the interoperability test suite derived in this paper. Since in general the time and resources required is proportional to the amount of execution of transitions, it is shown in this paper that the method is very efficient for the interoperability testing of protocols with limited time and resource comparing with validation. Moreover, the testing cost of interoperability could go down conspicuously while giving the same transition coverage as the validation one if we adopt a method (which need to work further) handling simultaneous open/close and message loss.

As a further work, one may think of extracting a test suite that has a couple of external inputs at once, by extending data structure used in our algorithm. In addition to that, further studies may be needed on testing FSM including primitives such as signal delay and data representation, and on testing protocols and systems in terms of service testing.

\section{REFERENCES}

[APRS 93] Arakawa, N., Phalippou, M., Risser, N. and Soneoka, T., "Combination of conformance and interoperability testing", Formal Description Techniques, V (C-10) M. Diaz and R. Groz (Eds.), Elsevier Science Publishers, 1993.

[AraS 92] Arakawa, N. and Soneoka, T., "A Test Case Generation Method for Concurrent Programs”, Protocol Test Systems, IV, Elsevier Science Publishers, 1992.

[GarR 95] Gary R. Wright, and W. Richard Stevens, "TCP/IP Illustrated, Volume2 The Implementation", Addison-Wesley Professional Computing Series, 1995.

[ISO 94] ISO/IEC JTC1 DTR-10000, "Information Technology - Framework and classification of international standard protocol", 1994.

[KanK 97] Kang, S., and Kim, M., "Interoperability Test Suite Derivation for Symmetric Communication Protocols", IFIP Joint International Conference on Formal Description Techniques (FORTE X) and Protocol Specification Testing and Verification (PSTV XVII), pp. 57-72, November 1997.

[LuBP 94] Luo, G., Bochmann G. And Petrenko, A., "Test Selection Based on communicating Nondeterministic Finite-State Machines Using a Generalized WpMethod", IEEE Transactions on S.E., Vol 20, No. 2, pp. 149-162, February 1994.

[RafC 90] Rafiq, O. and Castanet, R., "From Conformance Testing to Interoperability testing", Proceedings of the 3rd International Workshop on Protocol Test System, 1990.

[RFC 81] Postel, J. B., "Transmission Control Protocol", RFC 793, September 1981.

[Rich 91] W. Richard Stevens, "UNIX Network Programming”, Prentice Hall, 1991.

[SDT 97] "Telelogic SDT 3.2 Manuals", Telelogic, September 1997.

[ShiK 98] Shin, J., and Kang, S., "Interoperability Test Suite Derivation for the ATM/BISDN Signaling Protocol", Testing of Communicating Systems, Vol 11, Kluwer Academic Publishers, pp. 313-330, 1998. 
[VerB 94] Vermeer, G. S., and Blik, H., "Interoperability testing: Basis for the Acceptance of Communicating Systems", Protocol Test System, VI(C-19), Elsevier Science Publishers, 1994.

\section{APPENDIX 1. TEST SUITE FOR ATM/B-ISDN SIGNALING PROTOCOL.}

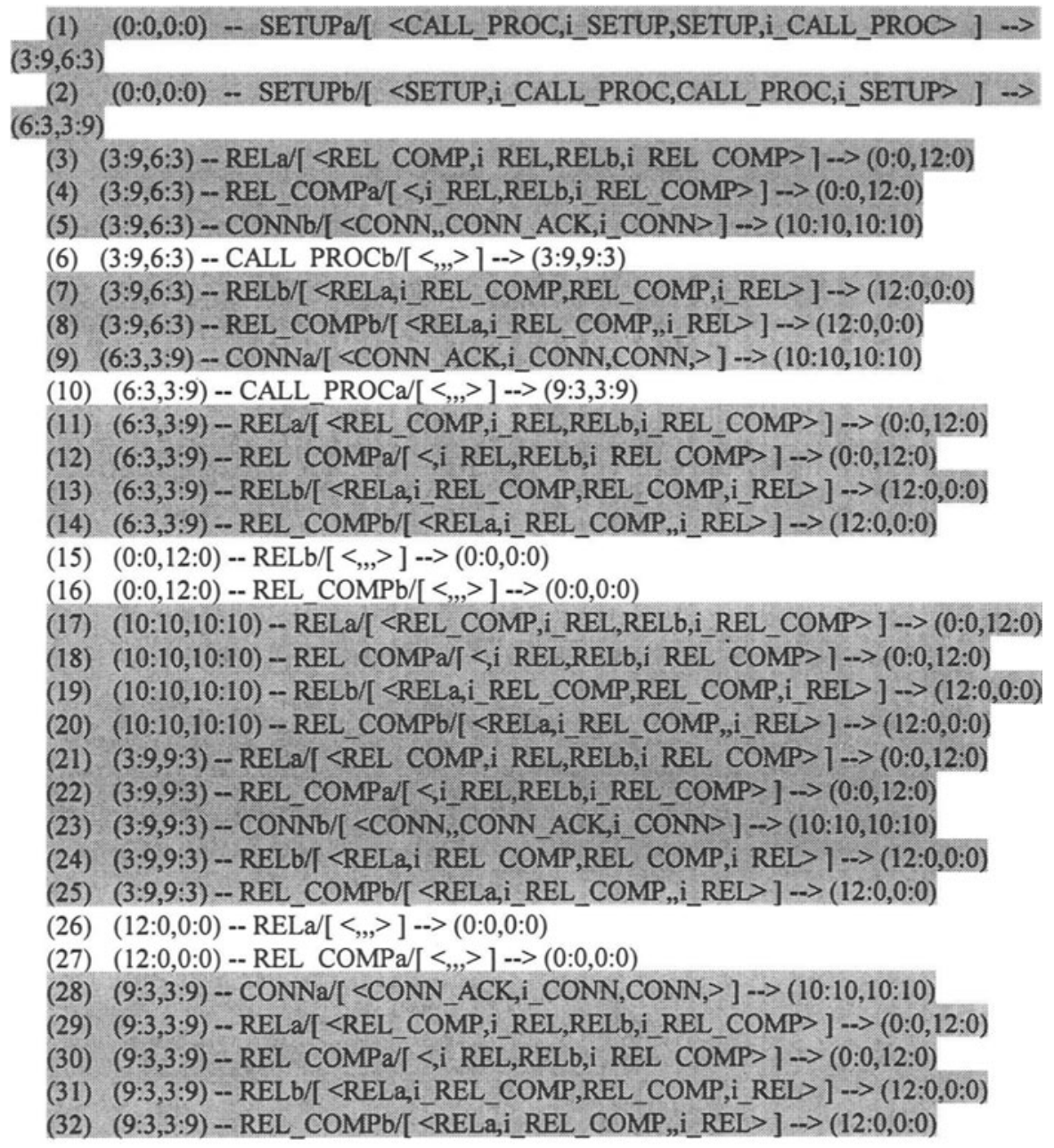

: interoperability test cases (Total number is 26) conformance

test cases (Total number is 6)

- A stable state and output symbols are represented as $\left(\mathrm{S}_{\mathrm{UNla}}, \mathrm{S}_{\mathrm{PNNla}}, \mathrm{S}_{\mathrm{UNIb}}, \mathrm{S}_{\mathrm{PNNIb}}\right)$ and [ $<a$ message from UNIa via interface A, a message from PNNIa via interface $\mathrm{C}$, a message from UNIb via interface B, a message from PNNIb via interface C> ], respectively. 


\section{APPENDIX 2. TEST SUITE FOR CONNECTION MANAGEMENT PART OF TCP.}
(1) (Closed,Closed) -- passive open $\mathrm{a} /[<, \ldots,>]-->$ (Listen,Closed)
(2) (Closed,Closed) -- active_open_a/[ $<$,i_SYN, $>] \rightarrow$ (SYN_Sent,Closed)
(3) (Closed,Closed) -- passive_open b/[ $<,,>]$-- $>$ (Closed,Listen)
(4) (Closed,Closed) --active_open_b/[<,,i_SYN $>] \rightarrow$ (Closed,SYN_Sent)
(5) (Listen,Closed) -- close $\mathrm{a} /[<$ closed,,, $>$ ] - $>$ (Closed,Closed)
(6) (Listen,Closed) - send_data_a/ $<$ i_SYN,, $>] \rightarrow$ (SYN_Sent,Closed)
(7) (Listen,Closed) -- passive_open_b/[ $<,,>]$--> (Listen,Listen)

(8) (Listen,Closed) - active open_b/[ <,i_SYN_ACK,i_SYN>, <established,established,i_ACK > ] - > (Estab,Estab)

(9) (SYN_Sent,Closed) -- close_a/[ <closed,,,> ] --> (Closed,Closed)

(10) (SYN Sent,Closed) -- passive open $b /[<,,>]-->$ (SYN Sent,Listen)

(11) (SYN_Sent,Closed) - $\quad$ active_open_b/[ <,i_SYN_ACK, i_SYN $>$, <established,established,i_ACK > ] $\rightarrow$ (Estab,Estab)

(12) (Closed,Listen) -- passive open $\mathrm{a} /[<,,>]$--> (Listen,Listen)

(13) (Closed,Listen) - $\quad$ active_open_all $<$, i_SYN,,i_SYN_ACK <established,i_ACK, established,>] $\rightarrow$ (Estab,Estab)

(14) (Closed,Listen) -- close b/[ <, closed, $>]$--> (Closed,Closed)

(15) (Closed,Listen) - - send_data_b/[<,,i_SYN $>] \rightarrow$ (Closed,SYN_Sent)

(16) (Closed,SYN_Sent) -- passive_open_a/[ $<,,>]$--> (Listen,SYN_Sent)

(17) (Closed,SYN Sent) - active_open_all <,i_SYN,i_SYN_ACK>, <established,i_ACK, established,>] $->$ (Estab,Estab)

(18) (Closed,SYN_Sent) -- close_b/[<,,closed,>] --> (Closed,Closed)

(19) (Listen,Listen) -- close a/[ <closed,,,> ] --> (Closed,Listen)

(20) (Listen,Listen) - $\quad$ send_data_a/[ <,i_SYN,i_SYN_ACK <established,i_ACK, established,>] $\rightarrow$ > (Estab,Estab)

(21) (Listen,Listen) -- close $\mathrm{b} /[<$, closed, $>$ ] --> (Listen,Closed)

(22) (Listen,Listen) - $\quad$ send_data_b/[ $\quad$,i_SYN_ACK,i_SYN $>$, <established,established,i_ACK > ] - > (Estab,Estab)

(23) (Estab,Estab) - close a/ $[<$, i FIN,,i ACK $>1 \rightarrow$ (FIN Wait-2,Close Wait)

(24) (Estab,Estab) - close_b/[<,i_ACK,i_FIN $>] \rightarrow($ Close_Wait,FIN_Wait-2)

(25) (SYN_Sent,Listen) -- close_a/[ <closed,,,> ] --> (Closed,Listen)

(26) (SYN Sent,Listen) -- close b/[<,, closed, $>$ ] - > (SYN Sent,Closed)

(27) (SYN_Sent,Listen) - $\quad$ send_data_b/[ $\quad$,i_SYN_ACK,i_SYN $\rangle$, <established,established,i_ACK > ] $\rightarrow$ (Estab,Estab)

(28) (Listen,SYN Sent) -- close a/[ $<$ closed,,,> $]$--> (Closed,SYN Sent)

(29) (Listen,SYN_Sent) - $\quad$ send_data_a/l $<$, i_SYN, i_SYN_ACK $>$, <established,i_ACK, established,>] $\rightarrow$ > (Estab,Estab)

(30) (Listen,SYN Sent) -- close $b /[<$, closed, $>]-->$ (Listen,Closed)

(31) (FIN_Wait-2,Close_Wait) - close_b/[ <closed,i_ACK,i_FIN $>] \rightarrow$ (Closed,Closed)

(32) (Close_Wait,FIN_Wait-2) - close_a/[ $<$,i_FIN,closed,i_ACK $>] \rightarrow($ Closed,Closed) 